\title{
Layout Techniques Coupled with Web2.0-Based Business Process Modeling
}

\author{
Philip Effinger ${ }^{1}$ and Gero Decker ${ }^{2}$ \\ 1 Wilhelm-Schickard-Institut, Eberhard-Karls-Universität Tübingen, Germany \\ ${ }^{2}$ Hasso-Plattner-Institut, Universität Potsdam, Germany \\ Effinger@informatik.uni-tuebingen.de, \\ Gero.Decker@hpi.uni-potsdam.de
}

\section{Introduction}

The most wide-spread notation for process models is the Business Process Modeling Notation (BPMN). With Oryx, we have a open-source modeling tool at hand that supports collaborative and web-based modeling of BPMN diagrams. Here, we show how automatic layout of diagrams can support the designer when starting to model a process in BPMN. We provide an automatic layout integrated into Oryx that computes a new layout for a given diagram considering BPMN drawing conventions, e.g. orthogonal edges, hierarchical structures, partitions, etc.

\section{Oryx - A Web2.0-Based Collaborative Graphical Editor}

Oryx (http://oryx-project.org) is an extensible framework for graphical modeling in the web browser. Using JavaScript and Scalable Vector Graphics (SVG), Oryx uses modern web technologies that realize a similar user experience like a classical modeling tool that runs on the desktop. The application is loaded into the browser whenever a graphical model is opened for editing.

In Oryx, each artifact is identified by a URL, so that models can be shared by passing references, rather than by exchanging model documents as email attachments. Oryx follows the Representational State Transfer (REST) architectural style, using the HTTP verbs GET, PUT, POST and DELETE for reading and updating models. This enables a highly scalable architecture, allowing for caching mechanisms at the protocol level.

The Oryx source code is available under an Open Source license and has become a widely used technology platform, especially in the Business Process Management (BPM) community. Here, process modeling using languages such as the Business Process Modeling Notation (BPMN) is a central activity.

\section{The Automatic Layout Algorithm and Integration into Oryx}

Our layout approach for support of automatic layout in ORYX is developed by extending previous works on layout techniques adoptable for BPMN [1, 2]. The layout is computed on BPMN diagrams that are based on a graphs. An automatic layout approach for BPMN-graphs has to support the drawing conventions that represent specific layout requirements of the BPMN notation. In our case, specfic layout requirements are:

D. Eppstein and E.R. Gansner (Eds.): GD 2009, LNCS 5849, pp. 417 4182010.

(C) Springer-Verlag Berlin Heidelberg 2010 


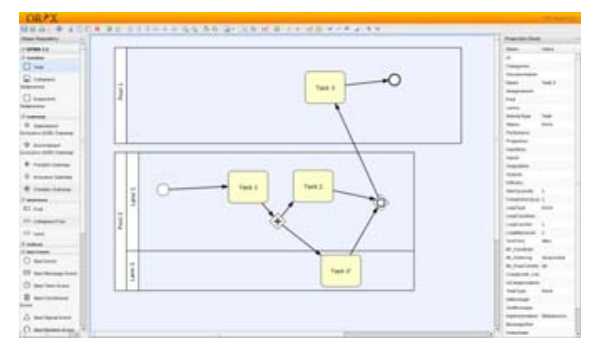

(a) A BPMN process diagram modeled by a human process designer. The Oryx user interface is completely browser-based and allows userfriendly drag-and-drop usability.

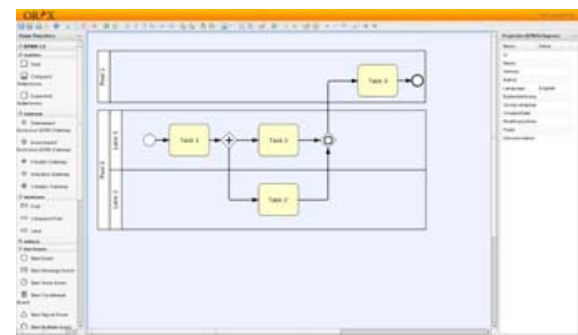

(b) The resulting process diagram of (a) after our approach is applied. The drawing conventions of BPMN are fulfilled.

- Elements have different sizes.

- We have to consider partitions, e.g. (collapsed/expanded) pools and swimlanes.

- Subprocesses may be nested and edges can start/end at a subprocess. This concept corresponds to graph clustering.

- Handle labels of pools, swimlanes, elements and edges.

Since BPMN-graphs are usually drawn using orthogonal routes for edges, we use an orthogonal layout approach for calculating the initial layout of a given BPMN-graph.

Our layout approach employs the implementation described in [3] that incorporates different constraints needed for the automatic layout of activity diagrams which are related to business process diagrams. The supported constraints include partitions (a generalization of swimlanes), clusters (subprocesses/groups) as well as a common workflow direction of edges which is especially important for such diagrams. Used techniques are based on Sugiyama's algorithm [4] and the Topology-Shape-Metrics (TSM) approach [5]. All layout requirements and drawing conventions demanded by BPMN models can be satisfied. Further details of this implementation can be found in [2].

For the integration of the layout implementations into Oryx, a wrapper was implemented in JAVA that offers interfaces for the connection to the JavaScript-based BPMNeditor Oryx and support of its diagram model.

\section{References}

[1] Effinger, P., Kaufmann, M., Siebenhaller, M.: Enhancing visualizations of business processes. In: Tollis, I.G., Patrignani, M. (eds.) GD 2008. LNCS, vol. 5417, pp. 437-438. Springer, Heidelberg (2009)

[2] Effinger, P., Siebenhaller, M., Kaufmann, M.: An Interactive Layout Tool for BPMN. In: BPMN 2009 - 1st International Workshop on BPMN, co-located with 11th IEEE Conference on Commerce and Enterprise Computing, CEC 2009 (2009)

[3] Siebenhaller, M., Kaufmann, M.: Drawing activity diagrams. In: Proceedings of ACM 2006 Symposium on Software Visualization, SoftVis 2006, pp. 159-160. ACM, New York (2006)

[4] Sugiyama, K., Tagawa, S., Toda, M.: Methods for visual understanding of hierarchical system structures. IEEE Transactions on Systems, Man, and Cybernetics SMC-11(2), 109-125 (1981)

[5] Tamassia, R.: On embedding a graph in the grid with the minimum number of bends. SIAM Journal on Computing 16(3), 421-444 (1987) 